\title{
Review on Recent Advances in Multiple Sclerosis and Related Disorders
}

\author{
Silvia N. Tenembaum ${ }^{*}$ and Garrahan JP \\ Department of Neurology, National Pediatric Hospital, Argentina \\ *Corresponding author: Silvia Tenembaum, Pediatric Neurologist Department of Neurology, National Pediatric Hospital, Argentina; E-mail: \\ silviatenembaum@gmail.com
}

Received date: Mar 31, 2014, Accepted date: Apr 25, 2014, Published date: Apr 30, 2014

Copyright: (c) 2014 Tenembaum S, et al. This is an open-access article distributed under the terms of the Creative Commons Attribution License, which permits unrestricted use, distribution, and reproduction in any medium, provided the original author and source are credited.

\begin{abstract}
The scientific world of central nervous system (CNS) neuroinflammatory disorders in general and multiple sclerosis (MS) in particular continues to expand, and 2013 has witnessed interesting advances in many aspects of research. In this review we will discuss research discoveries and results that have contributed to expand our knowledge in diverse areas of the MS field, including new environmental and genetic risk factors, new lines of treatment, and biomarkers of treatment response. Finally, new data on diagnostic biomarkers for neuromyelitis optica (NMO) have emerged, in addition to advances in NMO treatment.
\end{abstract}

Keywords: Multiple sclerosis; Environmental risk factors; Biomarker; Monoclonal antibodies; Neuromyelitis optica; MOG antibodies; AQP4 antibodies; MS treatment; NMO treatment

\section{Review}

Important advances in many aspects of multiple sclerosis (MS) research have been presented during 2013. In this review we will discuss results from a selected group of studies, evaluating their major contribution to MS research.

Epidemiological studies have shown that environmental exposures seem to be crucial for the development of MS. Vitamin D insufficiency, remote infection with

EBV, and exposure to cigarette smoke have already been proposed as environmental risk factors for both pediatric- and adult-onset MS. In 2013 new published data suggested that components of the daily diet could be considered additional risk factors for MS. Results from two independent studies suggest that high salt intake could trigger tissue inflammation and autoimmune disease. One study investigated environmental factors that directly influence TH17 cells, a newly identified population of interleukin (IL)-17-producing CD4 (+) helper $\mathrm{T}$ cells, highly pro-inflammatory cells with a key role in the development of experimental autoimmune encephalomyelitis (EAE), an animal model for MS [1]. The authors found that increases in sodium chloride concentrations markedly promoted the induction of murine and human TH17 cells. In animal models of MS, the TH17 cells generated under high-salt conditions displayed a highly pathogenic and stable phenotype characterized by the up-regulation of pro-inflammatory cytokines. The authors also found that mice fed with a high-salt diet developed a more severe form of EAE, with an increased number of TH17 cells peripherally induced and infiltrating the CNS. The second study showed that even a modest increase in salt concentration enhances TH17 cell differentiation in vitro and in vivo, accelerating the development of autoimmunity by inducing the expression of serum glucocorticoid kinase 1(SGK 1), which has a critical role in the regulation of IL-23R expression, reinforcing the TH17 phenotype [2]. According to these findings, a high-salt diet might represent an environmental risk factor for the development of autoimmune diseases by triggering TH17 cell differentiation and promoting tissue inflammation. Even though the causal association of these preliminary findings should be confirmed, they could be opening an interesting new therapeutic approach for patients with autoimmune disorders.

Results of a study published in 2013 introduced an additional risk factor for MS. The authors provided risk estimates that clearly show a strong association between increasing weight class and MS risk [3]. According to the data, overweight and obesity were associated with a significantly increased risk of developing MS or clinically isolated syndrome, particularly in girls. A potential mechanism for this association has been suggested in that obese people have lower levels of vitamin D metabolites than normal-weight people and decreased levels of serum 25-hydroxyvitamin D appear to increase MS risk. Furthermore, fat-related chronic inflammation may be involved. Adipose tissue produces and releases a variety of pro-inflammatory cytokines, including leptin, which promotes Th1 responses and reduces regulatory T-cell activity. Researchers have recently published data from a new study comprising two large populations of MS patients and controls, confirming the previously described association between obesity in early life and increased risk of developing MS [4]. Using a Swedish population-based case-control study as well as an American case-control study, the authors observed striking interactions between body mass index status and human leukocyte antigen (HLA)-DRB1* 15 allele, the gene most strongly associated with MS, with regards to MS risk.

Genetic research aimed at the identification of specific genes associated with pathways involved in the pathogenesis of MS continues growing, providing an overwhelming amount of new data. Multiple genome-wide association screens in MS have been completed in the past five years, expanding the list of risk loci and supporting the view that MS susceptibility results from multiple common allelic variants. In 2013, the International Multiple Sclerosis Genetic Consortium (IMSGC) revealed another 48 genetic polymorphisms contributing to risk of disease [5]. Although more than 100 genes have been described as involved with an increased risk of MS, many genetic polymorphisms could not be clearly associated with specific changes in protein expression. How these genes increase susceptibility to develop MS is a question which remains open. 
The biomarker research field in MS is very active as well. Although a great deal of progress has been made in this area, large gaps are still present between candidate, validated, and clinically useful biomarkers. Biomarkers in MS are expected to assist in the diagnosis, prediction of disease course, and identification of treatment response. An elegant and detailed review discussed the current state and future use of biomarkers in MS [6]. The challenges in developing and validating new tools to address benefits of a potential MS treatment were discussed. In particular, they emphasized how essential is the appropriate selection of useful clinical endpoints to identify reliable treatment-response biomarkers. Therefore, the authors proposed that changes in brain volume (atrophy), persistent black holes, T2 lesion volume, and retinal-nerve-fibre layer thickness, assessed by optical coherence tomography, were considered as surrogate endpoints to evaluate the treatment effect on neuroaxonal damage in progressive forms of MS. This is particularly interesting considering that a number of clinical investigators are currently searching for a treatment for progressive MS.

In the same line, a meta-analysis assessing the relationship between the size of treatment effect on brain atrophy and disability progression has been published in 2013 [7]. The authors found that treatment effects on disability progression correlated significantly with changes on brain atrophy and active MRI lesions. According to these results, the authors proposed MRI measures of brain atrophy as a surrogate outcome for treatment effect. In a second publication, the same group of researchers presented data indicating that treatment effect on relapses can be accurately predicted by the effect on MRI lesions [8]. Thus, the authors proposed to consider the use of MRI markers as primary endpoints in future clinical treatment trials for MS, particularly in specific situations such as trials testing generic or biosimilar versions of immunomodulatory drugs, or in pediatric trials testing drugs already approved for adult patients with MS.

Major advances in the treatment of MS have occurred during the last two decades. With the advent of monoclonal antibody (mAb) technology, several humanized mAbs were designed to target specific key molecules and immune cell subpopulations involved in the process leading to inflammation, demyelination, and axonal loss in MS. Daclizumab is a humanized mAb of the human IgG1 isotype that binds specifically to the a-subunit of the high- affinity IL-2 receptor (CD 25). Daclizumab works by masking the IL-2 binding site, resulting in the inhibition of several IL-2 dependent T-cell functions, including antigen- and mitogen-induced proliferation and cytokine secretion. Previous studies had shown that add-on daclizumab treatment in patients with active relapsing-remitting MS (RRMS) already treated with beta-interferon reduced the number of new or enlarged gadolinium contrast-enhancing lesions compared with interferon beta alone [9]. In addition, the authors found evidence suggesting that the beneficial clinical effect of daclizumab was at least partly associated with an expansion of a regulatory subset of CD56 bright natural killer (NK) cells. The potential protective role of NK cells in patients with RRMS had already been proposed in 2001, based on the observation of unusually high numbers of NK cells expressing IL-5 and IL-13 in the peripheral blood of patients during disease remission [10]. In 2013, results of a randomized controlled phase III trial (SELECT) using two doses of daclizumab high-yield process (HYP)(150 and $300 \mathrm{mg})$ in patients with RRMS were published [11]. The HYP form of the mAb has a different glycosylation profile of its amino-acids, resulting in decreased antibody-dependent cellular cytotoxicity. Daclizumab HYP treatment in the SELECT trial significantly reduced annualized relapse rate and disability progression compared to placebo with both doses [11]. Interestingly, authors found that circulating levels of CD56 bright NK cells were increased in patients treated with daclizumab HYP versus placebo, providing additional evidence that CD56 bright NK cells might mediate the therapeutic effects of this $\mathrm{mAb}$ in MS. In line with these findings, a phase 3 clinical trial of the expansion of CD56 bright NK cells as a response biomarker in daclizumab treatment is in progress. With regard to safety, daclizumab remained well tolerated overall, but serious adverse events attributed to this $\mathrm{mAb}$ were described11. Serious infections involving mainly the upper respiratory and urinary tract systems were slightly more frequent in the daclizumab group than in the placebo group ( $2 \%$ versus $0 \%$ ). However, most patients with serious infections resumed treatment after the infection resolved. Mild to moderate skin reactions were also reported in $20 \%$ of patients treated with daclizumab: allergic skin reactions, eczema, urticaria, folliculitis, erythema nodosum, pityriasisrosea, exfoliative dermatitis, alopecia, and psoriasis. Severe rashes consistent with a hypersensivity reaction occurred in $1 \%$ of daclizumab-treated patients in this trial [11]. One patient receiving daclizumab, who was recovering from a serious rash, died due to complication of a psoas abscess. With regard to serious adverse events affecting the liver, elevation of liver enzymes $\geq 5$ times greater than the upper limit of normal was more frequent in the daclizumab group compared to placebo ( $4 \%$ versus $<1 \%)$. In addition, there were four malignancies during the trial: two cases of cervix carcinoma (one in the placebo group and one in the daclizumab HYP $150 \mathrm{mg}$ group), and two cases of melanoma in the daclizumab HYP $300 \mathrm{mg}$ group [11]. Ofatumumab is a next-generation immunoglobulin (Ig) $\mathrm{G}$ subclass $1 \mathrm{mAb}$ that binds to $\mathrm{CD} 20$ on $\mathrm{B}$ lymphocytes and induces B-cell depletion via complement-dependent and antibody-dependent cell-mediated cytotoxicity.The first study exploring safety and efficacy of ofatumumab in RRMS has been recently published [12]. In this randomized controlled study, ofatumumab was associated with a profound selective reduction of $\mathrm{B}$ cells as measured by CD19 expression. This study provided Class II evidence that in patients with RRMS, ofatumumab does not increase the number of serious adverse events, and has a great impact on MRI activity decreasing new Tlgadolinium enhancing lesions, total enhancing T1 lesions, and new or enlarging T2 lesions, compared with placebo.

The identification of neuromyelitisoptica (NMO) as a nosological entity distinct from MS has improved dramatically our understanding of the disease [13], allowing for a better selection of specific treatment strategies and a substantial increase in NMO research. NMO is an autoimmune disorder with distinctive clinical, radiologic, and pathologic characteristics, and in most cases, a highly specific biomarker, a serum IgG autoantibody directed to aquaporin-4 (AQP4) is found. Nevertheless, some patients (10-30\%) remain seronegative for AQP4-Ab despite -fulfilling the international criteria of definite NMO [13]. Two independent research groups addressed the issue of AQP4 seronegativity. In one study, performed in a large French cohort of 87 NMO patients and 54 controls, and using the AQP4-M23 cellbased assay (CBA) as the best method to identify seropositivity, the authors could demonstrate that patients seronegative for AQP4-Ab (22 of 87) had specific demographic and disease-related features, distinct from seropositive NMO patients (65 of 87): an equal male/ female ratio, a Caucasian ethnicity, and an overrepresentation of simultaneous optic nerve and spinal cord involvement at first clinical event [14].

The second study included a large Japanese and Brazilian cohort of 215 pediatric and adult patients with a clinical diagnosis of 
Citation: Tenembaum SN and Garrahan JP (2014) Review on Recent Advances in Multiple Sclerosis and Related Disorders. J Neurol Neurophysiol S12: S2-016. doi:10.4172/2155-9562.S12-016

Page 3 of 3

definitive NMO or NMO spectrum disorder [15]. The presence of serum antibodies to AQP4 or to myelin oligodendrocyte glycoprotein (MOG) were tested using a cell-based assay. Most patients were seropositive for AQP4 antibodies (139/215; 65\%), with only $7 \%$ (16/215) with serum antibodies to MOG. Compared with AQP4 seropositive patients or patients who were seronegative for both antibodies, patients with MOG antibodies were more frequently male, had a more restricted phenotype (optic nerve more than spinal cord), more frequently bilateral simultaneous optic neuritis, more frequently had a single attack, the lower portion of the spinal cord more frequently involved, and demonstrated better functional recovery after an attack.

Advances in the treatment of NMO were also published in 2013. Treatment with eculizumab, a therapeutic mAb directed to complement protein C5, was evaluated in an open-label study [16]. Eculizumab treatment significantly reduced the frequency of disease exacerbations, and improved neurological disability measures in patients with aggressive forms of NMOSD.

\section{Acknowledgment}

Dr. Tenembaum has received lecture fees from Bayer Schering Pharma, Teva Pharmaceutical Ind., Biogen Idec., and Merck Serono. She received research support from Merck Serono.

\section{References}

1. Kleinewietfeld M, Manzel A, Titze J, Kvakan H, Yosef N, et al. (2013) Sodium chloride drives autoimmune disease by the induction of pathogenic TH17 cells. Nature 496: 518-522.

2. Wu C, Yosef N, Thalhamer T, Zhu C, Xiao S, et al. (2013) Induction of pathogenic TH17 cells by inducible salt-sensing kinase SGK1. Nature 496: 513-517

3. Langer-Gould A, Brara SM, Beaber BE, Koebnick C (2013) Childhood obesity and risk of pediatric multiple sclerosis and clinically isolated syndrome. Neurology 80: 548-552.
4. Hedström AK, Lima Bomfim I, Barcellos L, Gianfrancesco M, Schaefer C, et al. (2014) Interaction between adolescent obesity and HLA risk genes in the etiology of multiple sclerosis. Neurology 82 : 865-872.

5. International Multiple Sclerosis Genetics Consortium (IMSGC), Beecham AH, Patsopoulos NA Xifara DK, Davis MF, et al. (2013) Analysis of immune-related loci identifies 48 new susceptibility variants for multiple sclerosis. Nat Genet 45: 1353-1360.

6. Comabella M, Montalban X2 (2014) Body fluid biomarkers in multiple sclerosis. Lancet Neurol 13: 113-126.

7. Sormani MP, Arnold DL, De Stefano N (2014) Treatment effect on brain atrophy correlates with treatment effect on disability in multiple sclerosis. Ann Neurol 75: 43-49.

8. Sormani MP, Bruzzi P (2013) MRI lesions as a surrogate for relapses in multiple sclerosis: a metaanalysis of randomised trials. Lancet Neurol 12: 669-676.

9. Wynn D, Kaufman M, Montalban X, Vollmer T, Simon J, et al. (2010) Daclizumab in active relapsing multiple sclerosis (CHOICE study): a phase 2, randomised, double-blind, placebocontrolled, add-on trial with interferon beta. Lancet Neurol 9: 381-390.

10. Takahashi K, Miyake S, Kondo T, Terao K, Hatakenaka M, et al. (2001) Natural killer type 2 bias in remission of multiple sclerosis. J Clin Invest 107: R23-29.

11. Gold R, Giovannoni G, Selmaj K, Havrdova E, Montalban X, et al. (2013) Daclizumab high-yield process in relapsing-remitting multiple sclerosis (SELECT): a randomised, double-blind, placebocontrolled trial. Lancet 381: 2167-2175.

12. Sorensen PS, Lisby S, Grove R, Derosier F, Shackelford S, et al. (2014) Safety and efficacy of ofatumumab in relapsing-remitting multiple sclerosis: a phase 2 study. Neurology 82: 573-581.

13. Wingerchuk DM, Lennon VA, Pittock SJ, Lucchinetti CF, Weinshenker BG (2006) Revised diagnostic criteria for neuromyelitisoptica. Neurology 66: 1485-1489.

14. Marignier R, Bernard-Valnet R, Giraudon P, Collongues N, Papeix C, et al. (2013) Aquaporin-4 antibody-negative neuromyelitisoptica: distinct assay sensitivity-dependent entity. Neurology 80: 2194-2200.

15. Sato DK, Callegaro D, Lana-Peixoto MA, Waters PJ, de Haidar Jorge FM, et al. (2014) Distinction between MOG antibody-positive and AQP4 antibody-positive NMO spectrum disorders. Neurology 82: 474-481.

16. Pittock SJ, Lennon VA, McKeon A, Mandrekar J, Weinshenker BG, et al. (2013) Eculizumab in AQP4-IgG-positive relapsing neuromyelitisoptica spectrum disorders: an open-label pilot study. Lancet Neurol 12: 554-562.
This article was originally published in a special issue, entitled:

"Neurodegenerative Diseases: Symptoms and Therapeutics", Edited by Dr. Jin J Luo, Temple University School of Medicine, USA 